\title{
Intelligent Bot: A Cross Platform Mobile Application using Machine Learning Techniques and Bot Framework
}

\author{
Aniket Patil ${ }^{1}$, Bhushan Patil ${ }^{2}$, Shubham Salunkhe ${ }^{3}$, Suyog Naikwade ${ }^{4}$, Prof. P. M. Kamde ${ }^{5}$ \\ Department of Computer Engineering, Savitribai Phule Pune University, Sinhgad College of Engineering, \\ Vadgaon (Bk), Pune ${ }^{1,2,3,4,5}$
}

\begin{abstract}
Now-a-days to do the day-to-day tasks like searching on Internet, finding an optimized results, getting the right product, getting a minimum cost of any product, one needs to search a lot which leads waste of time and frustration. Every time it is not possible to get result as per requirement. An artificial intelligent personal assistant is needed to increase efficiency of doing such tasks. This project proposes a bot on cross platform mobile applications using machine learning algorithms. The purpose of bot is to recognize text, voice, image input, doing data analysis on the same and providing optimized result.
\end{abstract}

Keywords: Optimized result, Application Program Interface, K-Nearest Neighbor, Naive Bayes.

\section{INTRODUCTION}

In the past few decades, due to the increase in the accessibility of the World Wide Web, data is being generated on a large scale, every day. When the end user tries to access this data, the available variety of options makes it a difficult task to choose amongst products. In order to simplify this process, Intelligent Bot is designed. This Intelligent Bot, analyze and filter the preferences from past user experiences and generate Optimum Result for the target user.

Intelligent bot is utilized in a variety of areas: some popular applications include movies, music, news, books, research articles, search queries, social tags, and products in general. However, there are also recommender systems for experts, collaborators, jokes, restaurants, financial services, life insurance. Retrieval of data from search engine using Intelligent Bot based on the fundamental assumption that users are able to formulate queries that express their interests or information needs in term of intrinsic features of the items sought. In some cases, however, it may be difficult to identify suitable descriptors such as keywords, topics, genres, etc. that can be used to accurately describe interests. As the research grew in this area of information retrieval, many different strategies have been developed, tested and used for building accurate Optimized result. One of the most widely used and user friendly framework is bot framework. Also there are different algorithms like k-Nearest Neighbor algorithm, Naive Bayes Algorithm for filter user data, user metadata and also search result data according to user's requirement. In this paper, Xamarin cross platform which used for mobile application development is explained. This paper uses traditional machine learning techniques and implies few tweaks to it. A method of generating optimized result for users based on their browsing preferences along with their tagging preferences is introduced. The method proposed is based on generic dataset which is updated according to users search history. The users upon which the data was collected were fully aware of the contents and limits of the data usage. All users willingly provided the data and information used.

The remainder of the paper is organized as follows, Section 1 contains the Introduction. Section 2, discusses some of the related work in Xamarin, visual studio and optimization system, followed by Section 3 which explains the Survey Methodologies used in this research. Section 4, elaborates on the existing technologies in Optimize systems by analyzing few of the prominently used machine learning algorithms. Section 5 contains the data and results generated and the proposed idea. Finally, in Section 6, we conclude with conclusions and future work.

\section{METHODOLOGY}

An intelligent web-based chat bot depicts design and development of an intelligent searching chat bot. The paper presents a technology demonstrator to verify a proposed framework required to support such a bot (a web service). While a black box approach is used, by controlling the communication structure, to and from the web-service, the webservice allows all types of clients to communicate to the server from any platform. The service provided is accessible through a generated interface which allows for seamless XML processing; whereby the extensibility improves the lifespan of such a service. By introducing an artificial brain, the web-based bot generates customized user responses, aligned to the desired character. Questions asked to the bot, which is not understood is further processed using a third- 
party expert system (an online intelligent research assistant), and the response is archived, improving the artificial brain capabilities for future generation of responses. Xamarin cross platform is used for application development in all mobile operating system like Android, IOS, Windows etc. Using Microsoft Visual studio 2015. Mobile App provide user interface to access bot web services to get results and also to chat with bot. Using Xamarin Cross platform, Single code can use for developing app in all mobile OS.

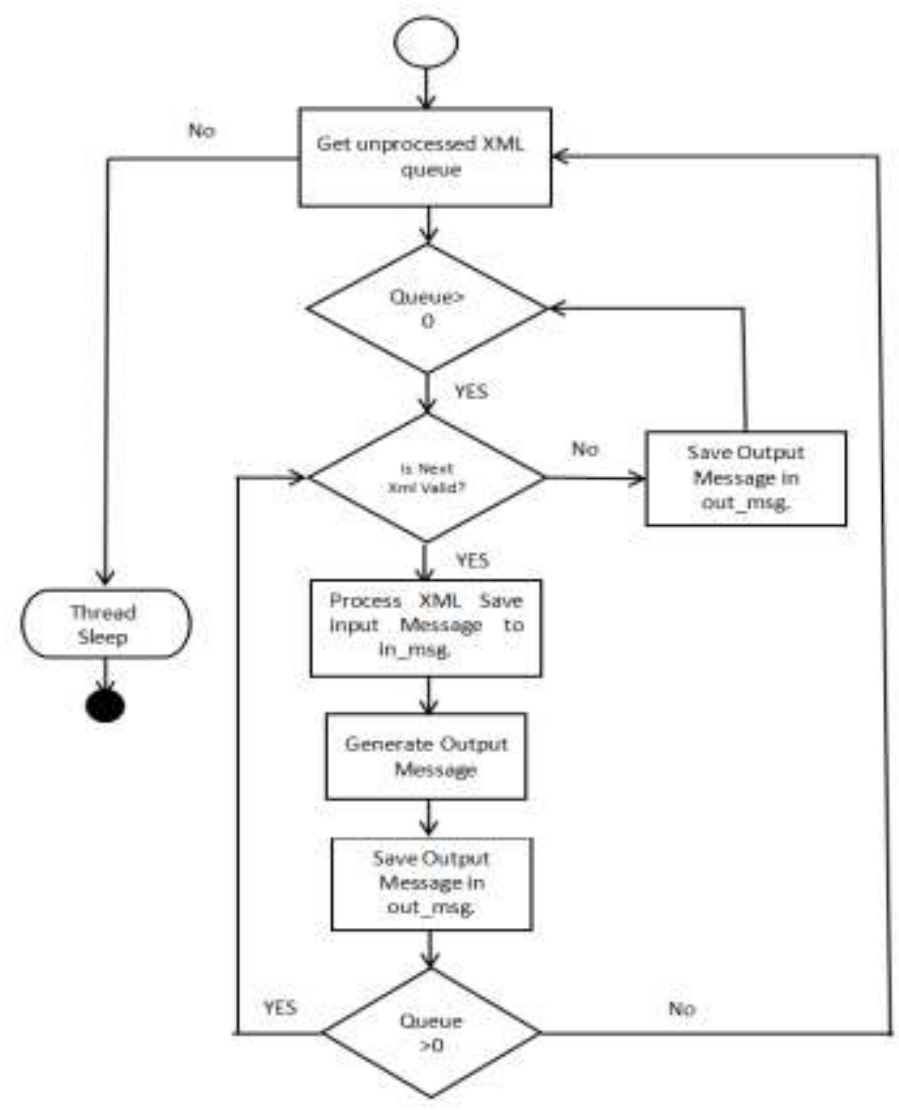

Bot Processing

Traditional machine learning algorithms like Naive Bayes and K-Nearest algorithms are used .The naive Bayes method (NB) is a simple Bayesian classifier based on Bayes' theorem from probability theory. In the WebDoc system, the stem forms of words occurring in the training documents were used as the features to represent each document. The basic steps in the naive Bayes method are as follows:

\section{Training:}

- Identify the individual stem words occurring in all the training documents in the training set.

- Generate the feature vector for each document in the training document set and store it along with the correct indexes in the knowledge base.

- Calculate the probability for each index.

\section{Testing:}

- Identify the individual stem words occurring in a given test document.

- Generate the feature vector for this document.

- Calculate the probability for this document given each index.

- Calculate the probability for each index in the set of indexes for this document and normalize it with Bayes' theorem, this value is the weight of this index.

- Select the indexes with a weight higher than a predefined threshold as the candidate indexes for this document.

The $\mathrm{k}$ Nearest Neighbor $(\mathrm{kNN})$ method is one of the most popular algorithm in clustering and data classification. The $\mathrm{kNN}$ algorithm founds to be performed very efficient in the experiments on different dataset. Classification of dataset is a wide span in research field, which includes many decision-theoretic approaches for predicting data. A data is typically denoted by a vector $(\mathrm{x} 1, \mathrm{x} 2, \cdots, \mathrm{xn})$ where $\mathrm{n}$ is the number of features. Therefore, each vector can be considered as one 
data in a $\mathrm{n}$ dimensional space, and belongs to some classes. Training and Testing are the two most vital steps in classification algorithm. Characteristic properties of the labeled training data will help to predict the class label of the testing data. There are so many classification algorithms, such as kNN.

The kNN rule proposed a new pattern is classified into the class with the most members present among the $\mathrm{k}$ nearest neighbor. kNN exhibits good performance and a less period of training time.

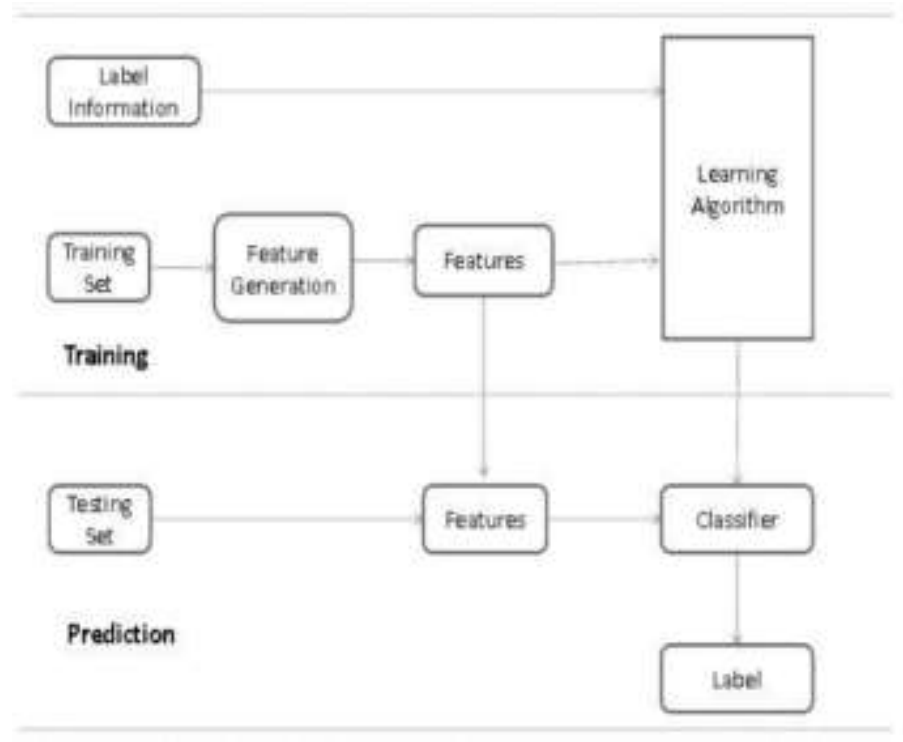

KNN and Naive Bayes algorithms use search history database, User's Metadata and search engine results database to classify data according to user's need and gives optimized result.

\section{PRIOR APPROACH}

In Existing searching system, user need to enter search query and search engine gives multiple results according to query. In those multiple results, many results are not useful to user and also to select appropriate result from set of multiple result is time consuming and hectic.

\section{OUR APPROACH}

The Machine learning techniques promotes conjunction of multiple machine learning algorithms. This conjunction methods can be applied to various domains over multiple databases to filter the data and thus to generate optimized result. Search results obtained from search engine is gathered and classifier algorithms used to minimize number of results of web documents. Also user can chat with bot. Due to this, these metadata can be used for increase in user's experience. By using training dataset, bot can give more effective results to user.

\section{CONCLUSION}

In this Paper, as part of initial stage, we developed mobile app to provide interface to user to access bot. We have analyzed machine learning algorithms and their functionalities. Though the execution of these algorithms are successfully tested in the singularity of their individual environments, we observe that they work best when hybridized together. In future scope, we can update user's query using users metadata and searching history. We can apply some algorithms to user's metadata and search history databases to understand user's choices.

\section{REFERENCES}

[1] Shemim Begum, Debasis Chakraborty, Ram Sarkar proposed "Data Classification Using feature Selection and kNN Machine Learning Approach". 2015 IEEE.

[2] Ming Xu, Chagzun Zue, "A Study and Application on Machine Learning of Artificial Intelligence", 2009 IEEE.

[3] S. J. du Preez1, Student Member, IEEE, M. Lall2, S. Sinha3, MIEEE, MSAIEE. ”An intelligent web-based voice chat bot", 2009 IEEE.

[4] Haichao Dong, Siu Cheung Hui and Yulan He, "Structural Analysis of Chat Messages for Topic Detection", 2006, Nanyang Technological University, Nanyang Ave, Singapore.

[5] Yong Wang, Julia Hodges, Bo Tang, “Classification of Web Documents Using a Naïve Bayes Method”, 2003 IEEE. 